\title{
Comment on "Some implications of the quantum nature of laser fields for quantum computations"
}

\author{
Wayne M. Itano \\ Time and Frequency Division, National Institute of Standards and Technology, Boulder, Colorado 80305
}

(Dated: January 16, 2003)

\begin{abstract}
A recent discussion of quantum limitations to the fidelity with which superpositions of internal atomic energy levels can be generated by an applied, quantized, laser pulse is shown to be based on unrealistic physical assumptions. This discussion assumed the validity of Jaynes-Cummings dynamics for an atom interacting with a laser field in free space, that is, when the atom is not surrounded by a resonant cavity. If the laser field is a multimode quantum coherent state, and the Rabi frequency is much greater than the spontaneous decay rate, then the total atomic decoherence rate is on the order of the spontaneous decay rate. With the use of a unitary transformation of the field states due to Mollow, it can be shown that the atomic decoherence rate is the same as if the laser field were treated classically, without any additional contribution due to the quantum nature of the laser field.
\end{abstract}

PACS numbers: 03.67.Lx, 42.50.Ct

The quantum dynamics of a two-level atom in free space interacting with a resonant, coherent, quantized electromagnetic field is important from the standpoint of pure physics and potentially for practical applications. For example, some proposed implementations of quantum computation depend on the ability to accurately generate arbitrary superpositions of two atomic states by means of applied, resonant fields. If the external field is considered to be classical, then the atomic dynamics (Rabi flopping), including decoherence due to technical imperfections in the classical driving field, can easily be calculated [see, e.g., Sec. 4 of Ref. [1]]. In addition, decoherence due to radiative decay of the atomic states has been considered [see, e.g., Secs. 4.2.1, 4.4.6.4 of Ref. [1]]. Conceivably, the quantum nature of the driving field might lead to additional decoherence.

A recent attempt 2] to extend the calculations of Rabi flopping in free space to the case of a quantized driving field used an inaccurate model, which is equivalent to a "reversed micromaser." That is, instead of an atom passing through a resonant cavity, an atom is intercepted by an electromagnetic field, confined to a region of space travelling at the speed of light. The context of this calculation was the necessity, in quantum computation, for high accuracy of quantum state control. Others have applied a more or less equivalent model to problems in quantum information processing [3]. In the "reversed micromaser" model, Fock states $|n\rangle$ apparently represent quantized field excitations confined to an imaginary box moving at the speed of light. While the atom is inside the field region, the atom-field state is presumed to follow Jaynes-Cummings dynamics [4]. In this model, a coherent laser pulse is represented by a superposition of moving Fock states $|\alpha\rangle=e^{-|\alpha|^{2}} \sum_{n=0}^{\infty}\left(\alpha^{n} / \sqrt{n !}\right)|n\rangle$. Jaynes-Cummings dynamics then lead to entanglement of the atom and field and to effective decoherence of the atomic dynamics when a trace is performed over the field degrees of freedom.

This picture is unrealistic and inaccurate for an atom in free space, since there the field is not confined by a cavity. The physical problem with the Jaynes-Cummings model in free space is that it assumes that there is only one mode of the field. All radiation emitted by the atom must go into that mode, and all radiation absorbed by the atom must come out of that mode. Thus, emitted radiation stays around and can be reabsorbed, and the absorption of radiation by the atom decreases the intensity of the applied field. The combination of these two effects leads to the complicated Jaynes-Cummings atomic dynamics, including the well-known collapses and revivals. The former effect (reabsorption of emitted radiation) does not occur in free space, because the emitted photon leaves the atom and does not interact with it again. The latter effect (a decrease in the applied field upon absorption of radiation by the atom) also does not occur in free space. It would correspond to a change in the laser pulse amplitude upstream from the atom. A change in the amplitude downstream does of course occur, due to interference with the coherent forwardscattered field. Radiation is emitted by the atom in a dipole (or other multipole) pattern into all modes of the field and also as coherent forward scattering. Because the electromagnetic field has all modes available to it, not just a single one, the atomic dynamics will differ from those predicted by the Jaynes-Cummings model.

The Jaynes-Cummings model makes an odd prediction, which might be called the "beam area paradox." The Jaynes-Cummings (or "reversed micromaser") model predicts that the decoherence of the atomic system scales inversely with the mean number of photons $\langle n\rangle$ in the laser pulse. If one keeps the intensity at the site of the atom constant, but increases $\langle n\rangle$ by increasing the cross-sectional area of the beam, the decoherence is predicted to decrease. This has the appearance of being a nonlocal effect of the presence or absence of the field at arbitrarily large distances from the atom. This result is more explicit in the work of van Enk and Kimble [3], where the beam area $A$ appears explicitly in, for exam- 
ple, Eq. (31), and where they state, "Decreasing the focal area $A$ will increase the amount of entanglement."

If the applied laser field is treated classically, but a phenomenological decay rate $\gamma$ for the upper level is included, one finds that the atomic decoherence rate is on the order of $\gamma$ if the field is strong. "Strong" here means that the time required for the atom to undergo an induced transition (Rabi-flop) is much less than the spontaneous lifetime of the upper state. The perhaps surprising fact is that no additional decoherence of the atomic system appears when the electromagnetic field is treated quantum mechanically, with the driving field being a quantized coherent state and the quantized vacuum field being present to induce spontaneous decay. This can be seen by making use of Mollow's unitary transformation [Eq. (2.8) of Ref. [5]]. It turns out that, in its effect on an atom, a quantum coherent field is equivalent to a classical ( $c$-number) field plus the quantum field, initially in the vacuum state. The proof of this result is given in detail in a textbook [6]. Since this result holds for a multimode coherent field, and not simply for an infinite plane wave, it is capable of describing a finite travelling laser pulse. This situation is similar to one that occurs in the calculation of the spectrum of resonance fluorescence. Mollow's original 1969 calculation of the spectrum simply assumed that the incident field was classical (i.e., $c$-number) 7]. In his 1975 calculation [5], he showed that this assumption was unnecessary and that the same spectrum is obtained if the incident field is treated as a quantized coherent state. It should be noted that this is an exact result, not one that is valid only in the large quantum number (classical) limit. The initially empty modes of the quantized field are eventually populated, but only at the timescale of the spontaneous decay. In addition, there is a coherent, forward-scattered, $c$-number field [see p. 1920 of Ref. [5] that, being $c$-number, does not lead to entanglement or decoherence of the atom. The fact that the total decoherence rate is of the order of $\gamma$ follows immediately from the fact that the probability that the field remains in the vacuum state is $e^{-\gamma t / 2}$, where $t$ is the time after the interaction has has started [Eq. (4.30) of Ref. [5]]. One can transform back to the ordinary frame, by using the inverse transformation, but this is not necessary for calculation of the atomic decoherence rate, since the unitary transformation involves only field operators and leaves the atomic state invariant. There is no "beam area paradox" in this treatment, since the interaction Hamiltonian depends (in the electric dipole approximation), only on the electric field at the position of the atom [e.g., Eq. (3.2a) of Ref. [5]]. Even if we go beyond the electric dipole approximation, the interaction still depends only on local properties, such as derivatives, of the field.

The main conclusion is that the decoherence of the atomic state upon application of a quantized, coherent field can be made as small as desired by making the interaction time sufficiently short compared to the spontaneous decay time. Of course, the intensity of the applied field must be high enough so that the desired operation, such as a $\pi$ transition, can be carried out in that time. The total decoherence rate is of the order of $\gamma$. There is no additional decoherence due to the quantum nature of the applied field, as long as it is in a coherent state. A similar conclusion holds for a Raman transition in a multilevel atom. That is, if the applied fields are coherent, then decoherence is the same as if the applied fields were classical and can be attributed to spontaneous emission [see, e.g., Sec. 4.4.6.4 of Ref. 1] for a discussion of decoherence for Raman transitions driven with classical fields].

I thank I. H. Deutsch for bringing to my attention the fact that he and A. Silberfarb have independently reached similar conclusions [8]. Some confusion might arise from the fact that, in the second paragraph of Sec. II of Ref. [8], Silberfarb and Deutsch state, regarding Refs. 2, 3], that "their conclusions are correct," but that "one must take great care to understand the regimes under which this formalism is applicable." In the rest of the paragraph, it is made clear that the formalism is not applicable to precisely the case under question, that is, to an atom in free space. They explicitly criticize the use of the JaynesCummings Hamiltonian [their Eq. (1)], which "falsely predicts the possibility of a single photon $2 \pi$ pulse in free space, whereby the photon is perfectly absorbed and reëmitted into the original mode." They further criticize the solutions for violating causality. In the following paragraph they trace the problems with causality to a "faulty quantization procedure."

In a recent preprint [Sec. III of Ref. 9]], GeaBanacloche modifies the arguments of Ref. [2] and claims that it is really the number of photons $n^{\prime}$ within a certain volume that is important for the decoherence, not the total number of photons $n$ in the laser pulse. That volume is given by the product of an effective cross section $\sigma_{\text {eff }}$ and the length of the laser pulse. The effective cross-section is $\sigma_{\text {eff }}=3 \pi / 2 k^{2}$, where $k$ is the wavenumber of the incident light. Even if this result has the right order of magnitude, as it appears to, the definition of $n^{\prime}$ seems to be arbitrary and seems to have been chosen to give the desired result.

This work was supported by the U. S. National Security Agency (NSA) and Advanced Research and Development Activity (ARDA) under Contract No. MOD7171.00 and the U. S. Office of Naval Research (ONR). This work is a contribution of NIST, an agency of the U. S. government, and is not subject to U. S. copyright. 
[2] J. Gea-Banacloche, Phys. Rev. A 65, 022308 (2002).

[3] S. J. van Enk and H. J. Kimble, Quantum Information and Computation 2, 1 (2002).

[4] E. T. Jaynes and F. W. Cummings, Proc. IEEE 51, 89 (1963).

[5] B. R. Mollow, Phys. Rev. A 12, 1919 (1975).

[6] C. Cohen-Tannoudji, J. Dupont-Roc, and G. Grynberg,
Atom-Photon Interactions, (Wiley, New York, 1992), p. 597.

[7] B. R. Mollow, Phys. Rev. 188, 1969 (1969).

[8] A. Silberfarb and I. H. Deutsch, quant-ph/0210056

[9] J. Gea-Banacloche, quant-ph/0212027 v2. 